Finisterra, XXXVI, 71, 2001, pp. 7-19

\title{
LES RESSOURCES DE TERRITOIRES ET LES TERRITOIRES DE RESSOURCES
}

\author{
Georges Benko ${ }^{1}$ et Bernard Pecqueur ${ }^{2}$
}

\begin{abstract}
Résumé - La recherche en géographie économique a connu un renouveau depuis les années 1990, on parle même d'une "nouvelle géographie économique». Mondialisation, métropolisation, formation des zones de libre-échange, commerce international, articulation du global et du local sont au centre des préoccupations de l'économie spatiale. Mais la mondialisation ne signifie pas homogénéisation des espaces. La notion du territoire est de retour dans les analyses économiques. Les territoires offrent des ressources spécifiques, intransférables, et incomparables sur le marché. Ces ressources spécifiques diversifient les espaces, et stabilisent les localisations des activités économiques. L'analyse des processus de révélation et de valorisation des ressources nouvelles, issues de la spécificité des espaces et des groupes humains qui y sont ancrés dans un contexte d'économie de services où domine la logique informationnelle, constitue probablement un des plus gros chantiers de l'analyse économique des années qui viennent.
\end{abstract}

Mots-clés: Géographie économique, économie territoriale, proximité, concurrence spatiale, ressources spécifiques.

Resumo - OS RECURSOS DE TERRITÓRIOS E OS TERRITÓRIOS DE RECURSOS. A investigação em geografia económica conheceu uma renovação na década de 1990 ; fala-se mesmo de uma «nova geografia económica». Mundialização, metropolização, formação das áreas de livre comércio, articulação entre o global e o local estão no centro das preocupações da economia espacial. Mas a mundialização não significa homogeneização dos espaços. A noção de território está de volta nas análises económicas. Os territórios oferecem recursos específicos, intransferíveis e incomparáveis no mercado. Esses recursos específicos diversificam os espaços e estabilizam as localizações das actividades económicas. Os actores locais e a política local desempenham um papel importante na constituição e na gestão dos recursos presentes no território. As perspectivas de criação de riqueza estão relacionadas com as capacidades dos grupos, quanto à organização e à elaboração de processos originais de geração de recursos. A análise dos processos de revelação e de valorização de novos recursos ligados à especificidade dos espaços e dos grupos humanos que estão presentes num contexto de economia de serviços, onde domina

1 Université Panthéon Sorbonne, 191, rue Saint-Jacques, 75005 Paris, France, E-mail : gb@univ-paris1.fr

2 Université Pierre Mendès France, IREPD, B.P. 47, 38040 Grenoble Cedex 9, France, E-mail : pecqueur@upmf-grenoble.fr 
a lógica informacional, constitui provavelmente um dos mais importantes domínios da análise económica dos próximos anos.

Palavras-chave: Geografia económica, economia territorial, proximidade, concorrência espacial, recursos específicos.

\begin{abstract}
THE RESOURCES OF TERRITORIES AND THE TERRITORIES OF RESOURCES. Research into economical geography has known a revival since the 1990s; we even speak of a «new economic geography». Globalisation, metropolisation, the formation of free-trade zones, international exchanges, the linking of the global with the local are all themes at the centre of the concern about spatial economy. But globalisation does not necessarily mean homogenisation of the spaces. The notion of territory reappears in economical analyses because territories offer the market resources that are specific, untransferable and incomparable. These specific resources render the areas diversified and stabilize the emplacement of economic activities. In the next few years, one of the main subjects of research in economic analysis will probably be the study of the process of revealing and developing new resources that emerge from the uniqueness of the areas and human groups in a context where information logic controls the service economy.
\end{abstract}

Key words : Economic geography, territorial economy, proximity, spatial competition, specific resources.

La recherche en économie régionale a connu un grand élargissement du champ de ses applications depuis les années 1980, les mutations sont considérables. La macroéconomie est de plus en plus ouverte vers l'espace et vers le territoire. La géographie économique se renouvelle à tel point qu'on parle d'une «nouvelle géographie économique» ou d'une «nouvelle géographie industrielle». Les travaux de Paul Krugman $(1991,1995)$ ont un impact sur les théories du développement régional. Commerce international, externalités, localisation industrielle sont à la base de son économie géographique. Il construit un modèle autour de l'intégration économique et développement des régions. MicHAEL PORTER analyse les stratégies concurrentielles et L'avantage concurrentiel des nations (1993). Mondialisation de l'économie, métropolisation, restructuration du système productif, économie d'archipel de Veltz (1996) ou États-régions de Ohmae (1996), les régions qui gagnent (Benko et Lipietz, 1992) et les régions qui perdent (CôTÉ, 1995), autant de mots clés et idées pour décrire un monde en mouvement accéléré et enrichir l'analyse économique.

\title{
I. CHANGEMENT D'ECHELLE EN ECONOMIE REGIONALE
}

Depuis les années 1970, on vit un phénomène de globalisation renforcé. Cela s'exprime à travers des échanges de différents types : informations, capitaux, biens et services, mais aussi par le déplacement de personnes. Les flux sont mondiaux.

La mondialisation désigne l'intégration croissante des différentes parties du monde sous l'effet de l'accélération des échanges, de l'essor des nouvelles 
technologies de l'information et de la communication, des moyens de transports, etc. Selon le point de vue où l'on se place, elle recouvre des processus bien spécifiques qui, pour les uns, prolongent des tendances anciennes, pour les autres, marquent une période nouvelle.

Pendant plusieurs siècles, les auteurs ont mis l'accent sur l'analyse de l'économie nationale et de son complément, l'économie internationale. Beaucoup trouvent normal, dans les années 1990, de privilégier la «mondialisation». Mais quelle mondialisation (ou encore globalisation)? Les images et les symboles sont nombreux, les interprétations variées.

Il y a d'abord la logique de l'histoire, le passé dans l'avenir. C'est une étape nouvelle dans le développement d'un processus pluriséculaire : le capitalisme... l'extension des «économies monde». Les économies monde ont été décrites par BRAUDEL dans son ouvrage monumental Civilisation matérielle et capitalisme (1967-1979). Il s'agit des aires de développement qui se sont succédé à partir du $\mathrm{XVI}^{\mathrm{e}}$ siècle au rythme des grandes découvertes et des innovations techniques. Leur dénominateur commun : l'existence de centres donneurs d'ordres (port ou capitale d'empire, comme Amsterdam ou Londres) organisant les régions périphériques en fonction d'une division du travail et des productions. S'appuyant sur les travaux de BRAUDEL, certains considèrent que la mondialisation parachève la constitution d'un système ou espace monde.

La globalisation touche aussi la société : c'est la convergence des modes de vie. Sur le plan culturel cela se traduit par la diffusion d'une culture universelle planétaire (à travers des marques emblématiques: Coca-Cola, Disney, les jeux Olympiques...) en parallèle à des processus «d'hybridation», de métissage (voire de "créolisation») des modes d'expression culturelle de dimension universelle qui puisent dans les cultures locales tout en étant réappropriés par ces cultures.

Récemment se sont développées des zones économiques qui ont précisément pour objectif et pour mérite de tenter de tirer le meilleur parti des interdépendances stratégiques entre divers domaines et divers pays dont les intérêts peuvent transitivement diverger. N'est-il pas ironique que le thème de la globalisation apparaisse au moment où se constituent trois grandes zones économiques: l'Alena en Amérique du Nord, l'Union européenne et une intégration économique de fait de l'Asie du Sud-Est dans le cadre institutionnel flexible et peu contraignant de l'Asean? On peut d'ailleurs interpréter le projet de la monnaie unique européenne comme exprimant la volonté des gouvernants de contourner l'inexistence d'un régime financier international cohérent et de peser éventuellement sur la négociation à venir sur un successeur au système de BRetTon Woods. Quant au «Grand Marché européen», le projet initial de JACQUES DELORS visait à consolider le style des institutions nationales, marquées par un État interventionniste et une couverture sociale étendue et à s'affranchir partiellement des aléas et des chocs venus du reste du monde. Le terme globalisation, popularisé par KENICHI OHMAE, devrait alors être remplacé par celui de «triadisation», que ce même Kenichi OHMAE a contribué à lancer! S'il n'est guère plus heureux, il est cependant plus respectueux des tendances à l'œuvre. 
Enfin, dernière ironie, l'aggravation dans les années 1970 et 1980 des déficits publics a incité les États à une décentralisation et à une régionalisation de la gestion des biens collectifs locaux, tels que les infrastructures de transports, l'éducation, la formation, voire même les aides au développement et à l'insertion sociale des chômeurs. On découvre alors que la densité des relations entre les acteurs locaux (entreprises, municipalités, universités, centres de recherche, syndicats) peut jouer un rôle déterminant dans la compétitivité de certaines activités industrielles et de services. Les districts industriels italiens semblent avoir leur équivalent dans le Bade-Wurtenberg tout comme dans certaines préfectures japonaises.

Un nouveau mot clé apparaît : la «glocalisation» ou l'articulation accrue des territoires locaux à l'économie mondiale. A l'origine, ce néologisme a été forgé par les managers japonais pour désigner un mode de gestion à la fois globale et locale de l'entreprise-réseau dans le cadre de l'économie mondialisée. En géographie, la notion de glocalisation est une manière de souligner la persistance d'une inscription spatiale de phénomènes économiques, la localisation des sites de production d'une multinationale dans les territoires.

Cet horrible néologisme "glocalisation" exprime à sa manière cette subtile synergie entre des institutions locales infranationales et la compétitivité appréciée sur les marchés internationaux... L'espace des régions ou encore celui des grandes agglomérations, permet, pour partie, la reconstruction de certaines des institutions économiques qui, lors des Trente Glorieuses, étaient exclusivement nationales.

La constitution d'une "économie d'archipel» (VelTz, 1996) ou de l'émergence à l'échelle de la planète d'un «archipel mégalopolitain mondial» organisé autour des mégalopoles nord-américaines et du Sud-Est asiatique.

Cette mondialisation de l'économie et du système d'information a provoqué la recomposition du monde, c'est-à-dire une révision des règles du jeu. On assiste à la formation de grandes régions économico-politiques (EuropeUnie, Alena, Mercosur, etc.), avec des méga-administrations. En Europe, on l'appelle communément «Bruxelles».

On se trouve face à un processus de régionalisation à travers la constitution de vastes zones de libre-échange, sur fond de «triadisation», autrement dit l'organisation de l'économie mondiale autour de trois grands pôles de développement majeur : l'Amérique du Nord, l'Union européenne et le Japon (la triade).

\section{ECONOMIE ET LE RETOUR DU TERRITOIRE}

Au cours des années soixante-dix et quatre-vingt on a découvert, comme nous l'avons déjà dit, que les relations de proximité entre les acteurs locaux peuvent jouer un rôle déterminant dans la compétitivité des activités économiques.

Les districts industriels - un concept introduit au début du siècle par l'économiste britannique Alfred MARShall - sont désormais de retour, aussi bien 
sur le terrain que dans les analyses. Ces lieux ont une caractéristique interne, «une personnalité régionale», comme disait jadis VIDAL DE LA BLACHE, l'un des pères de la géographie française. Leur spécificité découle d'une capacité, souvent héritée d'une culture ancienne, à négocier des modes de coopération entre capital et travail, entre grandes entreprises et sous-traitants, entre administration et société civile, entre banque et industrie, etc. Comme le note l'économiste Alain LipIETZ, en Emilie-Romagne ou dans le Bade-Wurtemberg, deux pôles de croissance économique, la stratégie du Parti communiste ou de la Démocratie chrétienne a consisté à rechercher le meilleur compromis social là où il était possible de le négocier, c'est-à-dire au niveau local : les hauts salaires, l'amélioration du cadre de vie, la qualification de la main-d'œuvre sont la contrepartie de la compétitivité des entreprises de ces régions.

Les politiques d'aménagement du territoire, à la charge du pouvoir central jusque dans les années quatre-vingt, ont également été déléguées aux collectivités territoriales. Le "développement local» se substitue désormais au développement "par le haut». "Il n'y a pas de territoires en crise, il y a seulement des territoires sans projet», déclarait ainsi en 1997 le ministre français de l'Aménagement du territoire. Cette approche est devenue incontournable aussi bien en économie qu'en politique. La prise en compte des facteurs locaux dans les dynamiques économiques apparaît aujourd'hui comme une évidence et une impérieuse nécessité. Il s'agit, somme toute, d'une préoccupation relativement récente, qui ouvre la voie vers la diversification des politiques économiques, sociales et culturelles.

On a beaucoup écrit aussi sur la nouvelle géopolitique de la production, qui a commencé à se cristalliser autour des liens entre les deux niveaux spatiaux d'activité économique - le local et le global. Dans la compétition mondiale, les régions et les localités sont désormais en situation de concurrence, mais cette dernière peut être analysée à deux niveaux. Le premier est celui de la maîtrise des coûts et de l'affectation optimale des facteurs de production. Les coûts de la main-d'œuvre, les prix de l'énergie, les taux d'intérêt et la fiscalité sont des variables qui, pour un grand nombre de productions, rendent les régions indifférenciées aux yeux des investisseurs. Qu'un jouet soit originaire d'une maquiladora à la frontière mexicaine ou d'une zone économique spéciale chinoise n'importe guère et seuls les coûts entrent en ligne de compte. En revanche, les spécificités territoriales jouent à plein dans d'autres domaines économiques. Dans ce cas, les régions ne sont guère substituables entre elles. Une différenciation durable des territoires, c'est-à-dire non susceptible d'être remise en cause par la mobilité des facteurs, ne peut ainsi découler que de leur spécificité reconnue. Même si l'on produit d'excellents vins mousseux en Californie, le champagne n'est pas un produit délocalisable. Sa valeur, comme celle d'autres productions est ancrée dans un territoire, elle est le résultat de ce que les géographes appellent un «milieu».

Notre monde «global» est ainsi une mosaïque composée d'une multitude de régions, de localités et de "pays», qui ne sont pas, loin s'en faut, nécessairement 
équivalents. Contrairement aux prédictions les plus sombres, les «territoires» avec leurs spécificités ne sont pas effacés sous les flux économiques de la mondialisation.

Ce constat est très évident et valorisé dans le secteur de l'agro-alimentaire. Mais, au-delà des produits d' «appellation contrôlée», dans le domaine de l'agroalimentaire, le même phénomène est observable dans les divers secteurs industriels (soit de hautes technologies, soit très spécialisés). Quand Rank Xerox cherchait une implantation européenne pour son centre de recherche, un des principaux critères de choix était la proximité d'établissements de recherche scientifique. Parmi les villes candidates (Barcelone, Munich, Toulouse) c'est finalement Grenoble qui a été retenue pour sa tradition (qui a commencé au milieu du XIX ${ }^{\mathrm{e}}$ siècle) dans le secteur de l'électricité et de l'électronique, pour son organisation industrielle. Notons l'existence, depuis 1947, de l'Alliance Universitaire de Grenoble, qui est un club régional de prospective entre universitaires et industriels, mais aussi la zone industrielle de haute technologie (la ZIRST), un mélange de la présence de grandes entreprises et de petites unités innovantes, entre autres, ce qu'on appelle "atmosphère industrielle» en référence à Marshall. Le quartier du Sentier à Paris continue à attirer le prêt-àporter, et celui du Faubourg Saint-Honoré la haute couture, pour leur spécificité. Les exemples sont multiples quand les facteurs décisifs de localisation sont hors marché (non quantifiables), et les éléments qualitatifs spécifiques à un lieu déterminent les choix des entreprises. La différence est un avantage comparatif.

Depuis les années 1980, les études se sont multipliées sous différentes formes autour de l'économie territoriale. Il suffit d'évoquer quelques mots clés comme développement local, milieu innovateur, district industriel, système productif localisé ou de penser à des théorisations nouvelles autour des coûts des transactions, réseaux, gouvernance, conventions, institutions, postfordisme. Plusieurs études présentent la synthèse de ses éléments (Pecqueur, 1989 et 1996 , BenKo et LiPIETZ,1998; BENKo, 1998).

Plus récemment, les nouvelles théories de croissance ont également contribué au progrès de l'économie géographique. L'idée que la croissance est localisée et dépend de facteurs propres à certains sites est à l'origine de nombreuses contributions empiriques récentes qui apportent un éclairage nouveau sur les mécanismes de la croissance.

Parallèlement, quelques chercheurs français (B. Pecqueur, Y. Lung, J.-B. Zimmermann, J.-P. Gilly, A. Rallet entre autres) organisent et orientent leurs travaux autour de la notion de "proximité», mais dans un autre esprit que Krugman, qui travaille aussi sur les externalités et sur l'agglomération. La proximité est définie en trois dimensions, la proximité géographique (espace géoéconomique, mais aussi proximité fonctionnelle), la proximité organisationnelle (l'expression de la séparation économique entre les agents, les individus, les organisations, etc., qui peut être appréhendée au plan technologique, industriel ou financier), et finalement la proximité territoriale, qui est l'interaction des deux formes de proximité. 
Cette approche met en valeur les proximités géographiques et organisationnelles au travers des institutions et de l'apprentissage collectif. Il ne s'agit pas de postuler le local, comme tend à le faire l'analyse en terme de milieu, mais de le déduire. Le territoire est un assemblage des pratiques et des représentations des agents économiques, mais il doit être aussi le résultat d'une démarche analytique et non son présupposé. La «proximité» est l'un des moyens de théoriser le territoire.

Cette école de pensée, nommée comme «école française d'économie de proximités», renouvelle l'économie industrielle régionale et se positionne dans la suite logique des travaux de la nouvelle géographie socioéconomique des années 1980, sur les milieux innovateurs, systèmes industriels localisés, districts industriels. C'est un prolongement qui permet de redynamiser et d'élargir les travaux pionniers de la décennie précédente.

Notons également que le terme de proximité est souvent évoqué depuis quelques années dans la recherche urbaine. La ville est l'expression par excellence d'une proximité organisée dans le but d'optimiser les interactions humaines. L'ouvrage dirigé par JEAN-MARIE HuRIOT (1998) analyse les différentes facettes de cette question, aussi bien au niveau théorique et méthodologique qu'empirique.

Globalisation ne signifie donc pas homogénéisation de l'espace mondial, mais au contraire différenciation et spécialisation. Des grands pôles se sont constitués, formant une économie en «oasis» ou en "archipels», c'est-à-dire un réseau des régions les plus dynamiques qui laissent derrière elles le reste du monde.

Les régions, ou encore mieux les territoires, sont ainsi devenues les sources d'avantages concurrentiels. Dans la section prochaine, nous allons voir comment on peut analyser les spécificités territoriales.

\section{LE TERRITOIRE ET LES RESSOURCES SPECIFIQUES}

L'analyse économique, de l'économie industrielle en particulier, accorde une place centrale à la compréhension des formes et mécanismes de la concurrence. La concurrence par les coûts et les prix constitue le noyau dur de l'analyse et l'hypothèse de référence des schémas de concurrence (concurrence pure et parfaite, concurrence oligopolistique). Des travaux empiriques récents (Mathis, Mazier et Rivaud-Danset, 1988) ont cependant montré au niveau des économies nationales que la maîtrise des coûts ne suffisait pas à assurer un commerce extérieur équilibré ou excédentaire.

$\mathrm{Au}$ niveau des entreprises, la maîtrise des coûts confère un avantage concurrentiel important, mais qui, seul, peut se révéler insuffisant. La capacité d'innover, de répondre rapidement aux variations de la demande en volume, de proposer des produits correspondants aux besoins spécifiques des demandeurs constitue aujourd'hui autant de variables essentielles des stratégies concurrentielles, parfois regroupées sous le terme quelque peu imprécis de «flexibilité» 
(Piore et Sabel, 1984).

L'accent mis sur les formes nouvelles de la concurrence, et notamment sur la concurrence par la différenciation de l'offre, peut conduire à proposer une typologie des facteurs de concurrence rendant compte de cette différenciation et qui ne soit pas nécessairement a priori marquée par un choix préalable exclusif du champ de cette concurrence : concurrence entre espaces économiques (pays ou régions) ou concurrence entre firmes. Ce type de réflexion se situe ainsi en amont méthodologique des analyses relatives aux facteurs de localisation des entreprises, qui distinguent plus qu'elles n'articulent les variables relatives aux stratégies des firmes et celles liées aux pays ou régions d'implantation (DunNing, 1988).

La typologie que nous proposons consiste à distinguer, d'une part, actif et ressources, d'autre part, à qualifier les actifs ou les ressources selon leur nature, génériques ou spécifiques.

Par actif, on entendra des facteurs "en activité», alors que par ressources il s'agira de facteurs à révéler, à exploiter ou encore à organiser. Les ressources, à la différence des actifs, constituent ainsi une réserve, un potentiel latent.

Des actifs ou des ressources génériques se définissent par le fait que leur valeur ou potentiel, est indépendante de leur participation à un quelconque processus de production (GAFFARD, 1990). Les actifs ou ressources sont ainsi totalement transférables, leur valeur est une valeur d'échange. Le lieu de cet échange est le marché. Le prix est le critère d'appréciation de la valeur d'échange, laquelle est déterminée par une offre et une demande à caractère quantitatif.

Les actifs spécifiques, d'après la définition que nous leur donnons, existent comme tels, mais leur valeur est fonction des conditions de leur usage. Alors qu'un actif générique est totalement transférable, un actif spécifique implique un coût irrécouvrable plus ou moins élevé de transfert. L'analyse en termes d'actifs spécifiques renvoie à celle des coûts de transaction développée par Williamson (1984). Comme l'observe GafFard (1990), cette analyse demeure cependant fondamentalement une théorie de l'affectation optimale des facteurs. D'après notre analyse, la différence entre actifs génériques et spécifiques est plus une différence d'intensité que de nature : c'est le degré de transférabilité ou si l'on préfère l'importance plus ou moins grande des coûts (irrécouvrables) de transfert, qui détermine la spécificité de l'actif. Hautement spécifique, un actif reste néanmoins transférable.

Selon notre perspective, les ressources spécifiques n'existent qu'à l'état virtuel et ne peuvent en aucun cas être transférées. Ces ressources naissent de processus interactifs et sont alors engendrées dans leur configuration. Elles constituent l'expression du processus cognitif qui est engagé lorsque des acteurs ayant des compétences différentes produisent des connaissances nouvelles par la mise en commun de ces dernières. Lorsque des connaissances et savoirs hétérogènes sont combinés, de nouvelles connaissances émergent qui ouvrent de nouvelles combinatoires. La création de technologie est ainsi le résultat d'un processus caractérisé par l'émergence de ressources spécifiques issues 
d'une dynamique cognitive synonyme d'un apprentissage interactif. L'avantage d'une telle définition de la création de technologie est qu'elle n'exclut pas de pouvoir configurer avec des termes adéquats des situations caractérisées par un transfert simple de connaissance d'où ne naît pas une technologie nouvelle.

Des actifs génériques et même spécifiques ne permettent pas à un territoire de se différencier durablement puisque, par définition, ils existent ailleurs et sont susceptibles d'être transférés. Une différenciation durable, c'est-à-dire non susceptible d'être remise en cause par la mobilité des facteurs, ne peut naître véritablement que des seules ressources spécifiques, lesquelles ne sauraient exister indépendamment des conditions dans lesquelles elles sont engendrées. L'importance est ainsi davantage de saisir ces conditions que de rechercher ce qui constituerait le potentiel identifiable d'un territoire. Ces conditions ne sauraient être définies de façon abstraite. Elles dépendent du contexte dans lequel s'inscrit le processus heuristique d'où naissent les ressources spécifiques.

Alors que les actifs spécifiques, parce qu'ils sont en activité et consacrés à un usage particulier plus ou moins déterminé, sont synonymes d'irréversibilité (Dosi et Metcalfe, 1991), les ressources spécifiques, pour ne pas être aléatoires - il n'y a pas de ressources spécifiques sans actifs spécifiques -, n’offrent de limites que celles liées à la créativité humaine.

Le tableau distingue quatre types de facteurs de concurrence spatiale. Nous chercherons ici à préciser chacun de ces types, en opérant toutefois un regroupement préalable selon qu'il s'agit de facteurs génériques ou spécifiques.

La dernière partie de cet article montrera comment des stratégies d'acteurs (entreprises et pouvoirs publics locaux) peuvent induire le passage d'une concurrence en termes de ressources à une concurrence en termes d'actifs (et inversement) génériques ou spécifiques.

TABLEAU I - Typologie des facteurs de concurrence spatiale

QUADRO I-Tipologia dos factores de concorrência espacial

\begin{tabular}{|c|c|c|}
\hline & GÉNÉRIQUE & SPÉCIFIQUE \\
\hline RESSOURCES & $\begin{array}{l}1 \\
\text { Facteurs de localisation non utilisés, } \\
\text { discriminés par les prix et le coût du trans- } \\
\text { port (calcul, optimisation). }\end{array}$ & $\begin{array}{l}4 \\
\text { Facteurs incommensurables et intrans- } \\
\text { férables dont la valeur dépend de l'orga- } \\
\text { nisation qui les crée. }\end{array}$ \\
\hline ACTIFS & $\begin{array}{l}2 \\
\text { Facteurs de localisation non utilisés, } \\
\text { discriminés par les prix et le coût du trans- } \\
\text { port (calcul, optimisation). } \\
\text { - allocation optimale des actifs }\end{array}$ & $\begin{array}{l}3 \\
\text { Facteur comparable dont la valeur est } \\
\text { liée à un usage particulier : } \\
\text { - coûts d'irréversibilité } \\
\text { - coûts de réaffectation }\end{array}$ \\
\hline
\end{tabular}




\section{Ressources et actifs génériques}

Le qualificatif de générique recouvre l'ensemble des facteurs traditionnels de définition spatiale (ou de localisation des activités économiques) discriminés par les prix (dont les coûts de transport) et qui font l'objet de la part des agents d'un calcul d'optimisation.

On illustrera les situations de ressources ou d'actifs génériques à l'aide de quatre exemples : la main-d'œuvre, le capital, la matière première et l'information. Ces quatre facteurs de production resteront à l'état de ressources génériques dans les cas suivants : la main-d'œuvre est non qualifiée et non utilisée. Il existe un stock de capital sous forme d'épargne potentielle mais non révélée ou thésaurisée. Les gisements de matière première existent mais ne sont pas exploités. L'information est disponible sous forme standard non utilisée. C'est le cas d'une banque de données, d'une encyclopédie ou d'un catalogue.

Ces ressources, en devenant des actifs, ne cessent pas pour autant d'être génériques. Elles ne changent pas de nature mais elles sont réalisées et acquièrent ainsi une valeur économique mesurée en prix. La main-d'œuvre reste non qualifiée mais est employée. Le chômeur non qualifié représente un actif et pas simplement une ressource car, même si son travail n'est pas réellement utilisé, il est présent et influe sur le marché du travail et donc sur le taux de salaire. Le capital prêt à s'investir se révèle et devient épargne. Cette épargne reste très liquide et ne peut donc être affectée qu'à des placements à court terme. La matière première est mise en exploitation. L'information, sous sa forme standard, est effectivement utilisée. Du point de vue métaphorique, l'encyclopédie est une ressource (elle se consulte), le livre est un actif (il se lit).

Les ressources comme les actifs génériques sont totalement dans le marché. C'est-à-dire que, pour les acquérir, il existe un prix de marché, ils sont totalement transformables, immédiatement disponibles. Pour ces actifs, il n'existe pas de contrainte géographique (hors de la matière première mais cette dernière est substituable). Ajoutons enfin que leur acquisition ne nécessite pas l'existence et l'activation de formes de coordination hors marché comme, par exemple, les réseaux.

\section{Actifs et ressources spécifiques}

La relation entre actifs et ressources, dans ce cas, est plus complexe que dans le cas précédent. Nous montrerons qu'il y a ici une différence de nature entre l'actif et la ressource. Le qualificatif de spécifique recouvre l'ensemble des facteurs, comparables ou non, dont la valeur est liée à un usage particulier.

Les actifs spécifiques ont un coût d'irréversibilité que l'on pourrait appeler aussi un "coût de réaffectation». Cela signifie que l'actif perd une partie de sa valeur productive dans le cas où il est redéployé vers un usage alternatif. En reprenant les illustrations utilisées plus haut, on dira que la matière

première ne peut pas avoir de caractère spécifique dans la mesure où sa valeur 
marchande n'est pas liée à son usage futur ni au contexte socioéconomique immédiat. Par contre la main-d'œuvre sera un actif spécifique dès lors qu'elle est qualifiée. Sa formation à une technique constitue un coût pris en charge partiellement hors de l'entreprise. Une délocalisation aurait, en partie, un coût de réaffectation pour l'entreprise utilisatrice de cette main-d'œuvre. De même, le capital devient actif spécifique lorsqu'il passe d'une forme d'épargne liquide à une forme de capital investi dans des équipements. Enfin, l'information devient, elle aussi, un actif spécifique lorsqu'elle s'élabore et s'organise en vue d'un usage particulier. Concrètement, on peut citer l'élaboration de logiciels très techniques concentrant l'information pour servir une production très pointue liée à des usages particuliers. Les actifs spécifiques ont en commun avec les ressources dans le cadre d'une organisation donnée.

Les ressources spécifiques ont une nature particulière par rapport aux trois autres ressources ou actifs des trois autres cadrans du tableau.

En premier lieu, ces ressources n'apparaissent qu'au moment des combinaisons des stratégies d'acteurs pour résoudre un problème inédit. Plus précisément, la formulation même du problème en même temps que sa solution font l'objet d'un processus heuristique marqué par des tâtonnements et des itérations successives.

En second lieu, ces ressources ne sont pas commensurables, ce qui signifie qu'on ne peut pas les exprimer en prix et qu'elles sont donc absolument non transférables.

En troisième lieu, la nature hors marché de ces ressources n'est pas incompatible mais complémentaire au marché. Ces ressources résultent d'une histoire longue, d'une accumulation de mémoire, d'un apprentissage collectif cognitif.

En dernier lieu, ces ressources, contrairement aux autres facteurs, sont indéfectiblement ancrées dans un territoire. Lémergence de telles ressources résulte en effet de règles, de coutumes, d'une culture élaborée dans un espace de proximité géographique et culturelle à partir d'une forme d'échange distincte de l'échange marchand : la réciprocité. Les caractéristiques de cette réciprocité au marché : l'échange est contraint (obligation sociale), il est différé dans le temps (le délai du retour attendu peut être très variable) et enfin ce retour n'est pas nécessairement monétaire, il peut être confiance, considération, reconnaissance ou savoir. La réciprocité comme terreau des ressources spécifiques est à la base des relations informelles qui composent une "atmosphère industrielle» au sens où l'entendait A. Marshall décrivant certaines concentrations des petites unités industrielles dans l' Angleterre ou dans l'Allemagne du début du $\mathrm{XIX}^{\mathrm{e}}$ siècle. Plus systématiquement, le sentiment d'appartenance à un lieu ou à un métier, la «culture d'entreprise» ou encore «l'esprit maison», font partie des ressources spécifiques (VEBLEN, 1899). La ressource spécifique d'un territoire, prise globalement, apparaît alors comme le résultat de processus long d'apprentissages collectifs qui aboutit à l'établissement de règles tacites. De telles règles ont la fonction de trier, de sélectionner et de hiérarchiser l'information, offrant ainsi à chaque acteur du territoire un espace d'intelligibilité. 


\section{CONCLUSION}

Dans cette période de mutation rapide dans laquelle les régulations d'un monde productif fordiste se font moins prégnantes, nous avons cherché à montrer qu'il existe de nouvelles modalités de création de ressources à l'échelle des stratégies locales d'acteurs.

De telles possibilités n'étaient pas ouvertes dans le contexte de croissance des «Trente Glorieuses» (des années de la période fordiste) dans l'économie de l'Europe d'après-guerre, où l'uniformisation des consommations était la règle et la condition de la croissance.

Aujourd'hui, le modèle est différent. Les perspectives de création de richesses tiennent aux capacités de groupes à s'organiser et à élaborer des processus originaux d'émergence des ressources. La globalisation, qui consiste essentiellement en l'interconnexion des marchés et qui crée des liens de cause à effet de plus en plus denses entre les acteurs économiques, produit en même temps des dynamiques et des procédures singulières de création de ressources. Ces stratégies visent à l'adaptation aux conditions nouvelles de la concurrence internationale, mais ouvrent en même temps des possibilités encore insuffisamment exploitées de créations d'activité.

C'est pourquoi il nous semble que l'analyse des processus de révélation et de valorisation des ressources nouvelles issues de la spécificité des espaces et des groupes humains qui y sont ancrés dans un contexte d'économie de services où domine la logique informationnelle, constitue probablement un des plus gros chantiers de l'analyse économique des années qui viennent.

\section{BIBLIOGRAPHIE}

BenKo, G. (1998) - La science régionale. Paris, PUF.

BenKo, G. (1999) - La mondialisation de l'économie n'est pas synonyme d'abolition des territoires. In CORdellier S., (ed)., Le nouvel état du monde: les 80 idées-forces pour entrer dans le $21^{e}$ siècle, Paris, La Découvert : 128-130.

Benko, G. ; Lipietz A. (eds.) (1992) - Les régions qui gagnent. Districts et réseaux : les nouveaux paradigmes de la géographie économique. Paris, PUF.

BenKo, G.; LipIETz A. (1998) - From the regulation of space to the space of regulation. GeoJournal, 44(4) : 275-281.

Benko, G. ; Lipietz A. (eds.) (2000) - La richesse des régions. La nouvelle géographie socioéconomique. Paris, PUF.

Colletis, G. ; Pecqueur B. (1993) - Les facteurs de la concurrence spatiale et la construction des territoires. Grenoble, Université de Grenoble II, Notes de Travail 11, IREPD.

Côté, S.; Klein J-L.; ProulX, M-U. (eds.) (1995) - Et les régions qui perdent...? Montréal, UQAM. 
Dosi, G.; Metcalfe, S. (1991)-Approches de l'irréversibilité en théorie économique. In Boyer, R. ; Chavance, B. ; Godard O. (eds.), Les figures de l'irréversibilité en économie, Paris, Edition EHESS: 37-68.

Dunning, J. H. (1988) - Explaining International Production. London, Unwin Hyman.

GAFFARD, J. L. (1990) - Economie industrielle ou de l'innovation. Paris, Dalloz.

Geneau de Lamarlière, I. ; Staszak ,J.-F. (2000) - Principes de géographie économique. Paris, Bréal.

Gilly, J.-P. ; Pecqueur, B.(1995) - La dimension locale de la régulation. In Boyer R., Saillard, Y. (eds.) Théorie de la régulation : l'état des savoirs, Paris, La Découverte, 304-312.

Huriot, J.-M. ; ed. (1998) - La ville ou la proximité organisée. Paris, Anthropos/Economica,

Krugman, P. (1991) - Geography and Trade. Cambridge, MA., MIT Press.

Krugman P., (1995) - Development, Geography, and Economic Theory. Cambridge, MA, MIT Press.

Mathis, J. ; Mazier, J. ; Rivaud-Danset, D. (1988) - La compétitivité industrielle. Paris, Dunod.

Ohmae, K. (1996) - De l'État-nation aux États-régions. Paris, Dunod.

Pecqueur, B. (1989) - Le développement local. Paris, Syros/La Découverte

Pecqueur, B. (ed.), (1996)-Dynamiques territoriales et mutations économiques. Paris, L'Harmattan.

Pecqueur, B. (1999) - Les processus de bifurcation de l'activité économique en milieu urbain : le cas de l'agglomération grenobloise. In Fontan J.-M., KLEIN J.-L., Tremblay D.-G., (eds.), Entre la métrpolisation et le village global, Sainte-Foy, Québec, Presses de l'Université du Québec :125-138.

Piore, M. J. ; SABel, C. F. (1984, 1989)-Les chemins de la prospérité. De la production de masse à la spécialisation souple. Paris Hachette.

Porter, M. (1986) - L'avantage concurrentiel. Paris, InterEditions.

Porter, M. (1993) - L'avantage concurrentiel des nations. Paris, InterEditions.

Rallet, A. (1993) - Choix de proximité et processus d'innovation technologique. Revue d'Economie Régionale et Urbaine, (3), 365-386.

Scotт, A. J. (2001) - Les régions et l'économie mondiale. La nouvelle géopolitique globale de la production et de la compétition économique. Paris, L'Harmattan.

Veblen, T. (1899) - The Theory of the Leisure Class. New York, The Mac Millan Company (tr. fr. Théorie de la classe de loisir. Paris, Gallimard, 1970).

Veltz, P. (1996) - Mondialisation, villes et territoires. Paris, PUF.

Williamson, O. E. (1975) - Markets and Hierarchies. Analysis and Antitrust Implications. New York, Free Press.

Williamson, O. E. (1984) - The Economics of Governance : framework and implications. Zeitschrift für die gesamte Staatswissenschaft, $\mathrm{n}^{\circ} 140$.

Zimmermann, J.-B. (1995) - Dynamiques industrielles : le paradoxe du local. In RAllet A., Torre, A. (eds.), Économie industrielle et économie spatiale, Paris, Economica:147-168.

ZimmermanN, J.-B. (1998) - Nomadisme et ancrage territorial : propositions méthodologiques pour l'analyse des relations firmes-territoires. Revue d'Économie Régionale et Urbaine, (2): 211-230. 